\title{
Three-Quantum Annihilation in Porous Vycor Glass
}

\author{
B. Jasińska, J. WaWryszczuk and R. Zaleski \\ Institute of Physics, Maria Curie-Sklodowska University \\ pl. Marii Curie-Skłodowskiej 1, 20-031 Lublin, Poland
}

Three methods of determination of $3 \gamma o$-Ps decay intensity are compared. The estimate of $3 \gamma$ fraction obtained from classical lifetime measurements can be distorted due to a higher absorption of the $\gamma$ quanta from the continuous spectrum of three-quantum decay, compared to two-quantum one.

PACS numbers: 36.10.Dr, 78.70.Bj

\section{Introduction}

During last years an interest in positron study of low- $\kappa$ and other porous materials has been awakened. These materials are characterized by very long $o$-Ps lifetimes, thus a large fraction of $o$-Ps annihilates into three $\gamma$ quanta. That fraction is

$$
f=\tau_{o \mathrm{Ps}} / \tau_{o \mathrm{Ps}(\mathrm{vac})},
$$

where $\tau_{o \mathrm{Ps}}$ and $\tau_{o \mathrm{Ps}(\mathrm{vac})}$ are $o$-Ps lifetime in the sample and in vacuum, respectively. Three-quantum annihilation studies become competitive to the traditional positron annihilation lifetime spectrometry (PALS), in particular in the relation to porous media and positron beam technique [1]. Below we compare the results of estimation of $3 \gamma$ intensity obtained by several classical methods. The measurements were performed with a set of porous Vycor glass samples in vacuum chamber (to eliminate ortho-para conversion in oxygen). Two samples of porous ordered silica (MCM-41) were also investigated. As a reference the nonporous Vycor glass was used.

\section{Results and discussion}

\subsection{Intensity of $511 \mathrm{keV}$ peak}

The fraction of $3 \gamma$ decays can be determined from the reduction of the intensity of the $511 \mathrm{keV}$ peak in ${ }^{22} \mathrm{Na} \gamma$ spectrum compared to that intensity in the 
sample of possibly low $3 \gamma$ content. If positronium is not formed, the fraction of $3 \gamma$ decays is $0.269 \%$. In our case as a standard the nonporous Vycor was chosen, in which the $3 \gamma$ fraction is estimated with an acceptable accuracy as $0.46 \%$. Even if the value of $3 \gamma$ fraction in the standard contains a certain systematic error, it can influence the final results by less than $0.2 \%$. The spectra have to be normalized to the same number of $\beta$ decays, i.e. to the same number of counts under the $1274 \mathrm{keV} \gamma$ peak. This method gives directly the fraction of $3 \gamma$ decays. As an example of the results we show in Fig. 1 the $3 \gamma$ fraction for a sample of

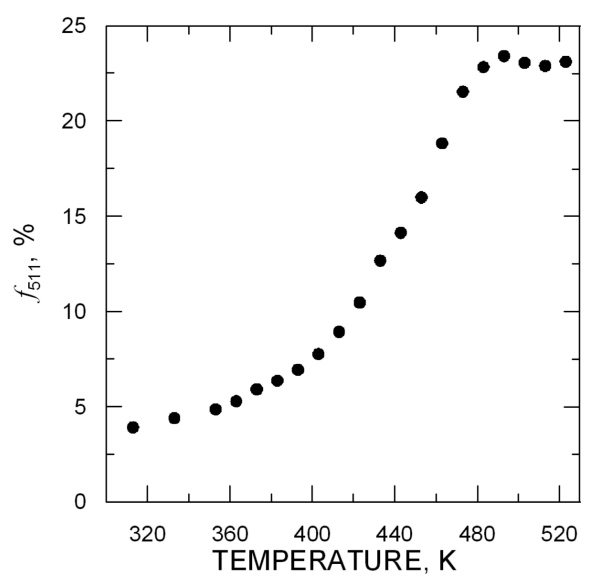

Fig. 1. The fraction of $3 \gamma$ o-Ps decays in MCM-41 raw sample as a function of annealing temperature.

MCM-41 ordered silica as a function of increasing temperature. Initial sample was the raw material with pores filled by the micelles of trimethyloctadecyl bromide. The rise in $f$ at highest temperatures means the removal of the organic template from silica structure and creation of empty pores. After pore evacuation and return to low temperatures the fraction $f$ remains high. In the literature one can find other parameter characterizing the $3 \gamma$ intensity, namely the ratio of net counting rate in the valley below the $511 \mathrm{keV}$ peak to that rate in the $511 \mathrm{keV}$ peak (or $1274 \mathrm{keV}$ peak) in ${ }^{22} \mathrm{Na} \gamma$ spectrum. Due to large Compton background from $1274 \mathrm{keV}$ under the valley region the statistical error in the measurements of this kind makes this method of little utility in classical measurements with radioactive positron source. Besides, at large $f$ the valley-to-511 peak ratio is not a linear function of $f$.

\section{2. $3 \gamma$ coincidence rate}

One can determine the rate of $3 \gamma$ decays by counting the triple coincidences in 3 coplanar counters inserted into the channels in a lead block with the sample chamber in the centre. The chamber was evacuated to $\approx 0.1 \mathrm{~Pa}$. When angular 
spacings between all counters are $120^{\circ}$, the energies of registered quanta from $3 \gamma$ decay are $341 \mathrm{keV}$ and the energy windows of single channel analysers are placed around that value. The background is estimated by moving one counter out of the plane determined by two other counters and sample. During the background measurement a $\mathrm{Ge}(\mathrm{Li})$ detector is inserted into the emptied channel and the energy spectrum (see Sect. 2.1) is measured at the same time. In order to determine the $3 \gamma$ fraction the triple coincidence rate should be compared to a standard sample. The "no-positronium" standard is not convenient, because in such case the $3 \gamma$ counting rate is very low and can contain a large error. On the contrary, the sample with large $3 \gamma$ fraction would be much more suitable.

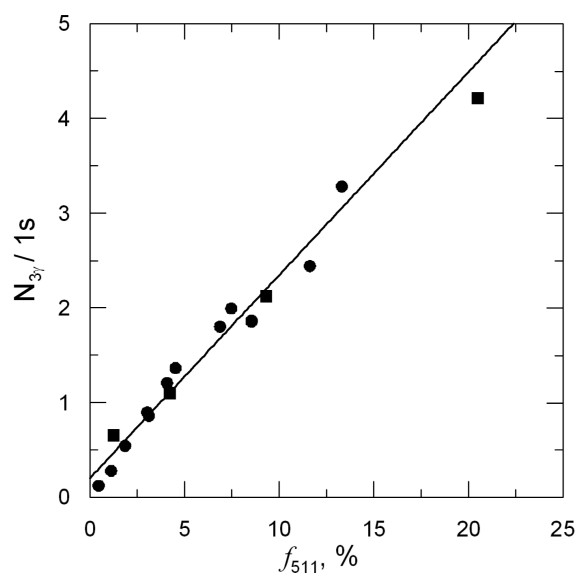

Fig. 2. The relation between the fraction $f$ determined from the relative decrease in $511 \mathrm{keV}$ peak intensity and the triple coincidence counting rate. Circles - Vycor glass, squares - MCM-41. Measurements at room temperature.

The counting rate in porous materials at our geometry, $\mathrm{NaI}(\mathrm{Tl})$ detectors $\varnothing 38 \times 40 \mathrm{~mm}$, and source activity (about $20 \mu \mathrm{Ci}$ ) reaches 3-4 coincidences per second. That allows us to collect good statistics in a reasonable time. Figure 2 shows the relation between $f$ estimated using the method described in Sect. 2.1 and triple coincidence rate. Note, that the data for both, Vycor and MCM-41, place roughly on the same curve.

\subsection{Lifetime spectra}

The fraction of $3 \gamma$ events can be determined from the intensities and lifetimes in the lifetime spectra:

$$
f_{\mathrm{LT}}=\frac{\left(1-\sum P_{i}\right)}{372}+\frac{3}{4} \sum \frac{\tau_{3 i}}{\tau_{3 o}} P_{i}
$$

where $P_{i}$ - the probability of Ps formation in $i$ th state, $\tau_{3 i}-o$-Ps lifetime in 
that state, $\tau_{3 o}$ - o-Ps lifetime in vacuum $142 \mathrm{~ns}$ (para-Ps does not participate in $3 \gamma$ events), 372 - the ratio of $2 \gamma$ to $3 \gamma$ probability.

The lifetime spectra were registered with a standard fast-slow setup and processed by LT programme [2]. It was assumed that the $o$-Ps component consists of two parts: the one with the lifetime of $1.33 \mathrm{~ns}$ (relative intensity $I_{3}$ ) representing $o$-Ps trapped in small free volumes in the amorphous glass structure, the other with the lifetime in the range of $7-100 \mathrm{~ns}$ (intensity $I_{4}$ ), belonging to $o$-Ps in the pores. Figure 3 shows $f$ estimate assuming in Eq. (2) $P_{i}=(4 / 3) I_{i}$ on the ordinate axis, and $f$ calculated from the decrease of $511 \mathrm{keV}$ peak on the abscissa.

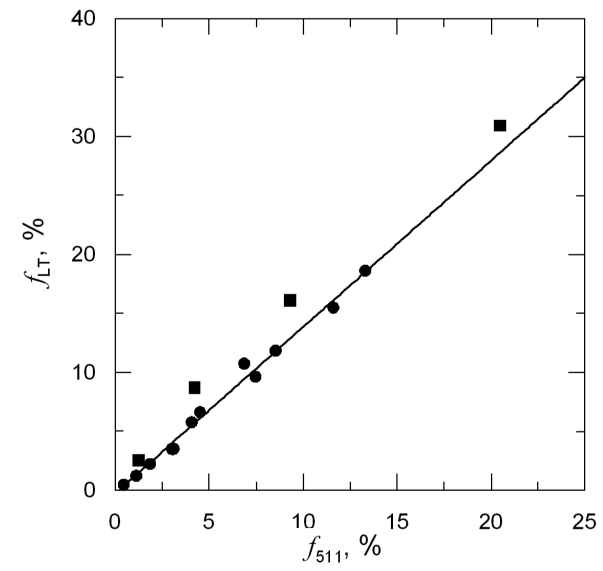

Fig. 3. The relation between fraction $f$ determined from the decrease in $511 \mathrm{keV}$ peak intensity and the same fraction calculated from PALS spectra. Circles - Vycor glass, squares - MCM-41 silica. Straight line is fitted to the Vycor data only. Measurements at room temperature.

It is clearly seen that the $3 \gamma$ fraction determined this way is overestimated by a factor of 1.4. The intensity of long-lived component in the lifetime spectrum is not a real measure of o-Ps formation probability. Its value depends strongly on the setting of energy window in the stop channel [3]. Low energy quanta from $3 \gamma$ decay are registered with a higher efficiency than $511 \mathrm{keV}$ ones. Setting the energy window in stop channel in a low energy range we receive an increased intensity of the component related to the events with high $f$. In our case the window covered $80 \%$ of the energy range, i.e. from 100 to $550 \mathrm{keV}$. The experimental points for MCM-41 locate still high (the lifetime spectra were taken on another spectrometer with a slightly different window setting).

In the positron annihilation experiments determining the value of $3 \gamma$ fraction it is very important to estimate the absorption of annihilation quanta in the sample and its holder. Differences in absorption coefficients for various samples and reference can distort the results. Those differences can appear due to different 
chemical compositions, densities, dimensions. In the study above all samples were of the same size and the same or similar elemental composition.

This work was partly supported by the State Committee for Scientific Research (KBN) grant No. 7 T09A 05521.

\section{References}

[1] Positron and Positronium Chemistry, Eds. Y.C. Jean, P.E. Mallon, D.M. Schrader, World Scientific, Singapore 2003, ch. 7.

[2] J. Kansy, Nucl. Instrum. Methods A 374, 235 (1996).

[3] C. Dauwe, Physicalia Mag. 12, 211 (1990). 